\title{
Risk and severity of motor vehicle crashes in patients with obstructive sleep apnoea/hypopnoea
}

\author{
A T Mulgrew, ${ }^{1}$ G Nasvadi, ${ }^{2}$ A Butt, ${ }^{1}$ R Cheema, ${ }^{1}$ N Fox, ${ }^{1}$ J A Fleetham, ${ }^{1,3}$ C F Ryan, ${ }^{1,3}$ \\ P Cooper, ${ }^{2}$ N T Ayas ${ }^{1,3,4}$
}

\section{See Editorial, p 481}

${ }^{1}$ UBC Hospital Sleep Disorders Program, Respiratory Division, University of British Columbia, Vancouver, Canada; ${ }^{2}$ Insurance Corporation of British Columbia, 151 West Esplanade, North Vancouver, Canada;

${ }^{3}$ Respiratory Division, Department of Medicine, University of British Columbia, Vancouver, Canada; ${ }^{4}$ Centre for Clinical Epidemiology and Evaluation, Vancouver Coastal Health Research Institute, Canada

\section{Correspondence to:}

Dr N Ayas, Diamond Health Centre, 2775 Laurel St, Vancouver, British Columbia, Canada V6R 1M9; najib.ayas@ vch.ca

Received 12 June 2007 Accepted 23 December 2007 Published Online First 30 January 2008

\begin{abstract}
Background: Obstructive sleep apnoea/hypopnoea (OSAH) appears to be associated with an increased risk of motor vehicle crashes (MVCs). However, its impact on crash patterns, particularly the severity of crashes, has not been well described. A study was undertaken to determine whether OSAH severity influenced crash severity in patients referred for investigation of suspected sleep-disordered breathing.
\end{abstract}

Methods: Objective crash data (including the nature of crashes) for 783 patients with suspected OSAH for the 3 years prior to polysomnography were obtained from provincial insurance records and compared with data for 783 age- and sex-matched controls. The patient group was $71 \%$ male with a mean age of 50 years, a mean apnoea-hypopnoea index (AHI) of 22 events/h and a mean Epworth Sleepiness Scale score of 10.

Results: There were 375 crashes in the 3-year period, 252 in patients and 123 in controls. Compared with controls, patients with mild, moderate and severe OSAH had an increased rate of MVCs with relative risks of 2.6 (95\% Cl 1.7 to 3.9), 1.9 (95\% Cl 1.2 to 2.8) and 2.0 (95\% Cl 1.4 to 3.0 ), respectively. Patients with suspected OSAH and normal polysomnography (AHI $0-5$ ) did not have an increased rate of MVC (relative risk $1.5195 \% \mathrm{Cl} 0.9$ to 2.5), $p=0.21$ ). When the impact of OSAH on MVC associated with personal injury was examined, patients with mild, moderate and severe OSAH had a substantially higher rate of MVCs than controls with relative risks of 4.8 (95\% Cl 1.8 to 12.4$), 3.0(95 \% \mathrm{Cl} 1.3$ to 7.0$)$ and 4.3 (95\% Cl 1.8 to 8.9), respectively, whereas patients without OSAH had similar crash rates to controls with a relative risk of 0.6 ( $95 \% \mathrm{Cl} 0.2$ to 2.5$)$. Very severe MVCs (head-on collisions or those involving pedestrians or cyclists) were rare, but $80 \%$ of these occurred in patients with OSAH $(p=0.06)$.

Conclusion: Patients with OSAH have increased rates of MVCs, and disproportionately increased rates of MVCs are associated with personal injury.

In a number of studies, drivers with obstructive sleep apnoea/hypopnoea (OSAH) have been found to have a higher rate of motor vehicle crashes (MVC) than drivers without OSAH, ${ }^{1-4}$ but it remains uncertain whether all patients with OSAH share this risk. It is not surprising that patients complaining of excessive daytime sleepiness have increased MVCs; indeed, drowsiness is a contributing factor in 10\% of all MVCs and $12 \%$ of near- miss MVCs. ${ }^{5}$ However, in previous studies, subjective sleepiness frequently showed no correlation with MVC rates. ${ }^{46-8}$ Furthermore, whereas many studies show a dose-response relationship between OSAH severity and MVC risk, ${ }^{39-11}$ an equal number do not. ${ }^{47812}$

It is difficult to obtain driving records on large numbers of patients and this has limited some of the previous studies. Many studies have used selfreported MVCs rather than validated MVCs (eg, from insurance or police records)..$^{61012-14}$ Some studies had relatively small numbers of patients with OSAH, and few studies included many drivers with milder degrees of OSAH. In addition, some studies did not have information about important potential confounders such as kilometres driven per week, body mass index (BMI), sedative medications, daily sleep duration and nocturnal shift work. Other studies have lacked a control group. ${ }^{8}{ }^{15-18}$ Perhaps most importantly, there was often little information provided concerning the severity of the MVCs in this population, with no distinction made between MVCs resulting in minor property damage and those causing injury or death. This may be important because MVCs related to sleepiness may be of a more severe nature as they are associated with lack of vigilance or even falling asleep at the wheel. ${ }^{19-21}$

We hypothesised that not only do patients with OSAH have an increased rate of MVC, but also that MVCs in patients with OSAH are more likely to be of a severe nature. To better understand the relationship between OSAH and MVC, we have calculated rates of validated MVCs categorised by severity in the 3 years prior to polysomnography (PSG) in a large population of patients with suspected sleep-disordered breathing and an ageand sex-matched control group. We also collected extensive information on potential confounders to allow multivariable regression analysis to determine the independent effects of OSAH on rates and severity of MVC.

\section{METHODS}

\section{Study sample}

Consecutive male and female adult subjects (>18 years) referred for overnight PSG for suspected sleep-disordered breathing were invited to participate in the study. All patients were referred to the Vancouver Acute Hospital Respiratory Sleep Disorders Program, the tertiary referral centre for sleep disorders in British Columbia, Canada. Patients with predominately non-respiratory sleep complaints (eg, narcolepsy, insomnia) are routinely referred to another group of physicians at the clinic and are therefore not strongly represented in our patient population. Patients were seen by one of five academic respirologists with a special interest in sleep-disordered breathing before PSG. Patients 
were excluded if they had symptoms of another sleep disorder known to cause daytime sleepiness (eg, periodic limb movement disorder), had another serious medical condition or overt psychiatric disease. Patients were also excluded if they were already established on treatment for OSAH at the time of referral or if they did not have a valid British Columbia driver's licence for the 3 years before PSG.

\section{Control group}

The control group was provided by ICBC from their database. Controls were individually matched to patients by age, sex and driver licence type and residence. The number of claims in the matched control group was checked against mean figures from the database to ensure the group was representative. No medical data were available for controls and their OSAH status and levels of subjective sleepiness were unknown.

\section{Techniques}

\section{Self-administered questionnaires}

Patients completed a number of surveys on the night of the PSG. These surveys included questions about work history, medical history, psychiatric history, sleep habits, health habits (including smoking, caffeine, alcohol use), sleep symptoms, daytime sleepiness (Epworth Sleepiness Scale $(E S S)^{22}$ ) and medications. For alcohol use, patients were asked to report their average use of beer (1 glass, bottle, or can), wine (4 oz glass) or liquor (1 drink or shot) over the past year (11 gradations of response ranging from never or less than once per month to more than 20 per day). Weekly alcohol use was calculated by summing the frequency of beer, wine and liquor use. Weekly caffeine use was estimated by asking patients to report how many cups of coffee with caffeine they used over the pervious year, using similar gradations.

\section{Polysomnography (PSG)}

Overnight PSG was performed using conventional instrumentation and analysis according to the recommendations on syndrome definition and measurement techniques published by the American Academy of Sleep Medicine. ${ }^{23}$ Sleep and its various stages were documented by standard electroencephalographic, electro-oculographic and electromyographic criteria. Apnoeas and hypopnoeas were recorded by oronasal flow cannulae attached to a pneumotachograph. Chest wall and abdominal movements were recorded using inductive plethysmography to document respiratory effort. Oxygen saturation was measured by pulse oximetry using a finger probe. Periodic limb movements were recorded from two surface electromyographic electrodes positioned 2-4 cm apart over the belly of the tibialis anterior muscle of each lower limb. Obstructive apnoeas were defined as the absence of airflow for $>10 \mathrm{~s}$ and obstructive hypopnoeas were defined as a $50 \%$ decrease in airflow or a clear but lesser decrease in airflow if associated with either a $>3 \%$ desaturation or an arousal in the context of ongoing respiratory effort. The complete record was scored manually for sleep stage, arousals, apnoeas and hypopnoeas.

\section{MVC information}

All motorists in the province of British Columbia, Canada are insured by a single crown corporation (the Insurance Corporation of British Columbia (ICBC)). Objective crash data for subjects in the 3 years before PSG were obtained from ICBC records including details regarding crash severity type. All MVCs are categorised in the insurance database into one of three severity groups: (1) crashes involving minor property damage $(<\$ 1000)$; $(2)$ crashes involving major property damage $(>\$ 1000)$ including damage to both the driver's car and third party damage; and (3) crashes involving personal injury. More detailed descriptions of crash location and nature were also available (eg, serious crashes involving head-on collisions, cyclists or pedestrians).

All patients were matched with an individual control for age, sex, type of licence and driving experience. Patients and controls were also matched by postal region since people residing in the same geographical area are more likely to experience similar travel patterns associated with urban/rural residency and socioeconomic status.

\section{Data analysis}

All data are reported as mean (SD) unless otherwise noted. A p value of 0.05 was considered statistically significant. Patients were divided into four groups of OSAH severity based upon the apnoea-hypopnoea index (AHI): ${ }^{23}$ normal PSG (AHI $\leqslant 5$ events/ h), mild OSAH (AHI $>5$ and $\leqslant 15)$, moderate OSAH (AHI $>15$ and $<30$ ) and severe OSAH (AHI >30). Patients were also divided into four quartiles of subjective sleepiness severity depending on their ESS.

Crash rates were compared for patient severity groups according to AHI or ESS. MVCs occur in a Poisson distribution, so Poisson regression in a forward step model was used to assess the effect of potential confounders. Differences between rates of MVC in patients and controls were assessed for significance using the McNemar test for discordant pairs.

\section{RESULTS}

\section{Patient characteristics}

A total of 783 patients were identified and matched with individual controls drawn at random from the ICBC database. Most of the patients were men (71\%) with a mean (SD) age of 49.9 (11.6) years. The mean (SD) AHI was 22.6 (21.9) events/h, mean (SD) BMI was $31.8(10.3) \mathrm{kg} / \mathrm{m}^{2}$, mean (SD) ESS was 10.1 (5.3) and the mean (SD) kilometres driven per week was 234 (208). The demographic characteristics of the patients are shown in table 1.

\section{MVC characteristics}

MVC data were collected for a period of 3 years extending back from the date of PSG. In the 3 -year period there were 375 crashes, 252 in patients and 123 in controls. In the patient group there were 94 crashes causing minor property damage, 83 crashes causing major property damage and 74 crashes causing injury compared with 48, 52 and 23 in the control group. Crash breakdowns are shown in table 2 and fig 1 .

\section{Relationship between MVC and OSAH severity}

The study group was stratified into groups according to sleep apnoea severity. The rate of MVC over the 3 years before PSG evaluation was calculated for each severity group. Compared with controls, patients with OSAH had a significantly increased rate of MVC with a relative risk ranging between 1.9 and 2.6 (table 2), whereas patients who did not have OSAH (AHI 0-5) were not at increased risk compared with controls with a relative risk of 1.5 (95\% CI 0.9 to $2.5, p=0.21)$. A withinpatient analysis showed that the overall crash rate increased as OSAH severity increased from a mean (SD) of $0.24(0.5)$ crashes/patient/3 years in patients with an AHI of $0-5$ to 0.35 
Table 1 Mean (SD) demographic characteristics of study patients

\begin{tabular}{llllll}
\hline & $\begin{array}{l}\text { Total } \\
(\mathbf{n}=\mathbf{7 8 3})\end{array}$ & $\begin{array}{l}\text { AHI 0-5 } \\
(\mathbf{n}=\mathbf{1 4 0})\end{array}$ & $\begin{array}{l}\text { AHI 6-15 } \\
(\mathbf{n}=\mathbf{2 3 5})\end{array}$ & $\begin{array}{l}\text { AHI 16-30 } \\
(\mathbf{n}=\mathbf{2 0 6})\end{array}$ & $\begin{array}{l}\text { AHI }>\mathbf{3 0} \\
(\mathbf{n}=\mathbf{2 0 2})\end{array}$ \\
\hline Age $($ years $)$ & $49.9(11.6)$ & $46.9(11.2)$ & $49.7(11.0)$ & $49.6(11.4)$ & $52.7(12.3)$ \\
$\mathrm{BMI}\left(\mathrm{kg} / \mathrm{m}^{2}\right)$ & $31.8(10.3)$ & $31.2(19.9)$ & $31.1(6.4)$ & $31.4(6.4)$ & $33.1(7.7)$ \\
Sex $(\%$ male $)$ & 71.1 & 59.7 & 66.5 & 72.3 & 82.8 \\
$\mathrm{AHI}$ & $22.6(21.9)$ & $2.4(1.5)$ & $9.8(2.9)$ & $21.4(4.2)$ & $53.6(20.9)$ \\
ESS & $10.1(5.2)$ & $10.2(5.1)$ & $9.9(5.3)$ & $9.3(5.1)$ & $10.9(5.2)$ \\
$\mathrm{km} /$ week driven & $234.3(208.3)$ & $195.3(201.9)$ & $237.7(211.2)$ & $223.3(201.7)$ & $266.6(206.9)$
\end{tabular}

AHI, apnoea-hypopnoea index; BMI, body mass index; ESS, Epworth Sleepiness Scale.

(0.7) crashes/patient/3 years in patients with an AHI $>30$ (fig 1), but this difference was not significant.

Also shown in table 2 are rates of MVC subdivided into three severity categories. MVCs causing minor property damage were increased in patients with OSAH compared with controls whereas MVCs involving major property damage were not. Within patients there is no evidence of a dose-response relationship between OSAH severity and MVC causing property damage. The presence of OSAH caused a disproportionate increase in rates of more severe MVCs. Compared with controls, patients with OSAH had a 3.0-4.8-fold increased rate of MVC causing injury whereas those without OSAH (AHI 0-5) had similar MVC rates to controls (fig 2). MVCs involving personal injury represented $25.6 \%$ of the total. Within the patient group there appeared to be a dose-response relationship between OSAH severity and rate of MVC with injury, with the proportion of this type of MVC rising as OSAH severity increased (fig 3). In patients with an AHI of $0-5$, MVCs involving personal injury accounted for $9 \%$ of crashes compared with $37 \%$ in patients with an AHI $>30$. Compared with patients with an AHI of $0-5$, the relative risk of MVCs causing injury in patients with severe OSAH (AHI >30) was $6.1(95 \%$ CI 1.6 to 18.1). Patients with mild OSAH (AHI 5-15) had a 4.9fold (95\% CI 1.5 to 16.1) increased risk of MVCs involving personal injury compared with those with an AHI of 0-5.

MVCs of an extremely serious nature (head-on collisions or those involving pedestrians or cyclists) were rare, with only 10 in the 3-year period, $80 \%$ of which were in patients with OSAH. No serious MVCs occurred in patients without OSAH (AHI 05 ), three occurred in patients with mild OSAH (3.6\% of total accidents in this group), two in patients with moderate OSAH (3.1\% of total accidents) and three in patients with severe OSAH ( $4.2 \%$ of total accidents). Only two crashes of this nature occurred in the control group. The mean (SD) rate of MVCs of an extremely serious nature was $0.01(0.1) / 3$ years in patients compared with $0.002(0.05) / 3$ years in controls $(p=0.057)$.

\section{Relationship between MVCs and subjective sleepiness}

$\mathrm{MVC}$ rates were calculated according to the degree of subjective sleepiness (quartiles) as measured by the ESS. There was no significant difference in MVC rates among the quartiles (fig 4). When subsets of MVC (minor property damage, major property damage or personal injury) were examined, the ESS had no effect on the crash rate. In addition, the ESS did not influence the rate of extremely serious crashes.

\section{Multivariable modelling}

Multivariable Poisson regression was performed to control for the effect of a number of potential confounders. The following variables were included in the model (table 3): AHI group, ESS, BMI, gender, age, kilometres driven and use of alcohol, sedatives or caffeine. After controlling for confounders, the presence of OSAH increased the rate ratio for any motor vehicle crash to 1.22 (95\% CI 0.92 to 2.01 ), but this was not statistically significant $(p=0.12)$. Of note, BMI and kilometres driven were both significant in the model with $p$ values of 0.008 and 0.003 , respectively. However, the presence of OSAH increased the rate ratio for crashes causing personal injury to 3.67 (95\% CI 1.30 to 10.3, $\mathrm{p}=0.014)$. The kilometres driven was also nearly significant in the model $(p=0.053)$. Sex was not significantly associated with increased MVC rates in patients.

In control subjects a simplified regression model including only age and sex was used. Male sex was associated with an increased rate of any MVC $(p<0.001)$ whereas age was not a significant predictor in the model for all crashes. Increased age was associated with a significantly reduced rate of more serious crashes.

Table 2 Relationship between severity of crashes and severity of AHI

\begin{tabular}{|c|c|c|c|c|c|c|c|c|}
\hline & \multicolumn{2}{|c|}{ AHI $0-5(n=140)$} & \multicolumn{2}{|l|}{ AHI 6-15 $(n=235)$} & \multicolumn{2}{|c|}{ AHI $16-30(n=206)$} & \multicolumn{2}{|l|}{ AHI $>30(n=202)$} \\
\hline & Patients & Controls & Patients & Controls & Patients & Controls & Patients & Controls \\
\hline Total MVC & 33 & 22 & 83 & 32 & 64 & 34 & 71 & 35 \\
\hline $\begin{array}{l}\text { MVC with minor property } \\
\text { damage }\end{array}$ & 17 & 10 & 33 & 15 & 20 & 12 & 24 & 11 \\
\hline Relative risk (95\% CI) & $1.7(0.8$ to 3.4$)$ & & $2.2^{*}(1.2$ to 4.0$)$ & & $1.7(0.8$ to 3.3$)$ & & $2.2^{*}(1.1$ to 4.4$)$ & \\
\hline $\begin{array}{l}\text { MVC with major property } \\
\text { damage }\end{array}$ & 13 & 7 & 27 & 12 & 22 & 15 & 21 & 18 \\
\hline MVC with personal injury & 3 & 5 & 24 & 5 & 21 & 7 & 26 & 6 \\
\hline Relative risk $(95 \% \mathrm{Cl})$ & $0.6(0.2$ to 2.5$)$ & & $4.8 \dagger(1.8$ to 12.4$)$ & & $3.0^{*}(1.3$ to 7.0$)$ & & $4.3^{* *}(1.8$ to 8.9$)$ & \\
\hline
\end{tabular}

$\mathrm{AHI}$, apnoea-hypopnoea index; MVC, motor vehicle crash.

Crashes are categorised into one of three severity groups. Relative risks are shown between patients and controls.

${ }^{*} \mathrm{p}<0.05 ;{ }^{* *} \mathrm{p}<0.005 ; \mathrm{p}<0.001$ (McNemar test). 


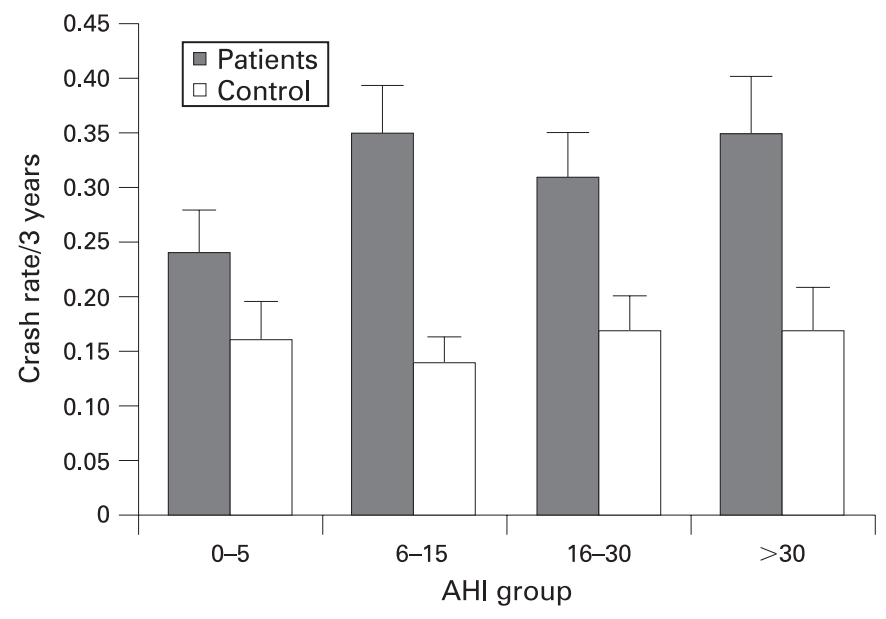

Figure 1 Mean crash rates over 3 years for patients according to severity of obstructive sleep apnoea/hypopnoea and their respective controls. AHI, apnoea-hypopnoea index. Error bars represent standard error of the mean.

\section{DISCUSSION}

Compared with controls, patients with OSAH had an increased rate of MVC. Importantly, the relative risk was much greater for crashes associated with personal injury than for those involving property damage only. Our large sample allowed us to perform regression analysis for a number of confounding variables. After multiple regression, the presence of OSAH did not significantly increase the odds of unselected MVCs but was associated with a more than threefold increase in the odds of MVCs associated with injury.

To our knowledge, the greater impact of OSAH on crashes involving injury has not previously been demonstrated. However, studies looking specifically at severe MVCs have been performed and support the concept that decreased vigilance and/or falling asleep at the wheel are associated with a more severe spectrum of crashes, perhaps because sleepinessrelated crashes may be related to failure to brake/reduce speed, driving off the road or higher speeds. ${ }^{19}{ }^{24}$ In our study, any degree of OSAH was associated with an increase in the MVC rate. This contrasts with the findings of George and associates ${ }^{3}$ who noted an increased MVC rate only in patients with an AHI $>40$, but is consistent with data from Young and colleagues ${ }^{8}$ which showed an adjusted odds ratio of 4.2 for MVCs in patients with an AHI of 5-15. We did not find significant differences in MVC rates when patients with mild OSAH were compared with patients with severe OSAH. Interestingly, OSAH had little effect on MVCs involving major property damage. Although insurance databases commonly include severity cut-offs for property damage, our impression was that inclusion in this category was more influenced by the cost of the vehicle than by the actual crash impact. It is therefore not unexpected that OSAH did not influence this category of MVC.

We adjusted for several other confounding variables including age, kilometres driven, sex and substance use. MVC rates were related to kilometres driven and BMI. Although we identified a significant association between alcohol use and injury-associated crashes, we found no relationship between MVCs and sedatives or stimulants. There is no clear reason why BMI should have increased the rate of unselected MVCs in our regression analysis, but there are potential explanations. First, obese patients may have difficulty moving easily in the drivers'

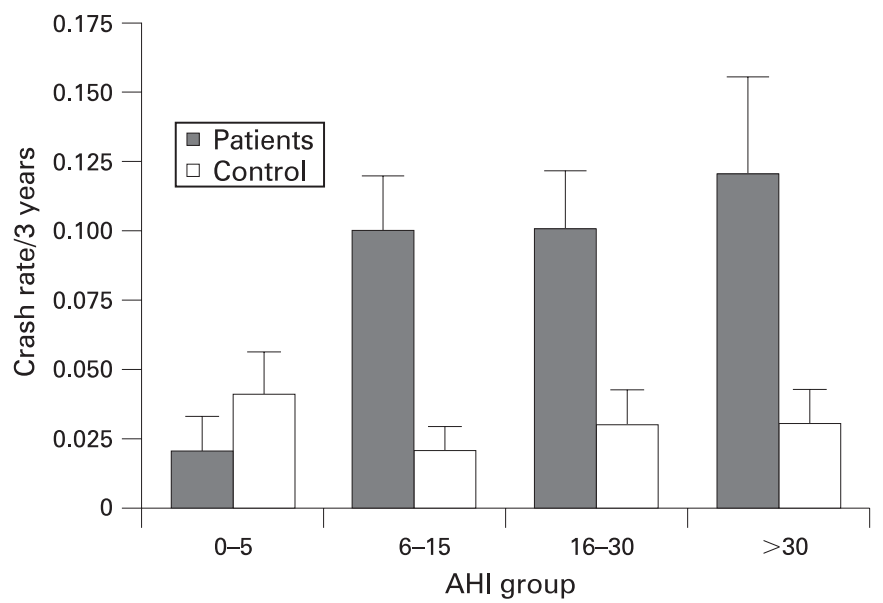

Figure 2 Mean rates of crashes causing injury over 3 years for patients grouped by severity of obstructive sleep apnoea/hypopnoea and their respective controls. AHI, apnoea-hypopnoea index. Error bars represent standard error of the mean.

seat, leading to reduced ability to see traffic approaching from behind or the side. Second, adipose tissue increases levels of inflammatory mediators (such as tumour necrosis factor) which may result in reduced vigilance independently of sleep apnoea severity. ${ }^{25}$ Third, obesity may be a marker of subtle sleepdisordered breathing events that may not be captured by the standard definitions of AHI as assessed by PSG. Given that the severity of sleep apnoea (as assessed by AHI) does not correlate well with sleepiness and did not correlate well with crash risk in our study, we may need to search for other metrics of sleepdisordered breathing that could be better indicators of crash risk. ${ }^{26}$

Another interesting finding from our regression analysis was that sex did not significantly influence the rate of MVCs among patients with OSAH. The MVC rate in women with OSAH was 0.27 crashes $/ 3$ years compared with 0.36 crashes $/ 3$ years in men. In contrast, our regression analysis showed sex to be a significant predictor of MVCs in controls $(0.11$ crashes/ 3 years in women and 0.18 crashes $/ 3$ years in men). OSAH appears to

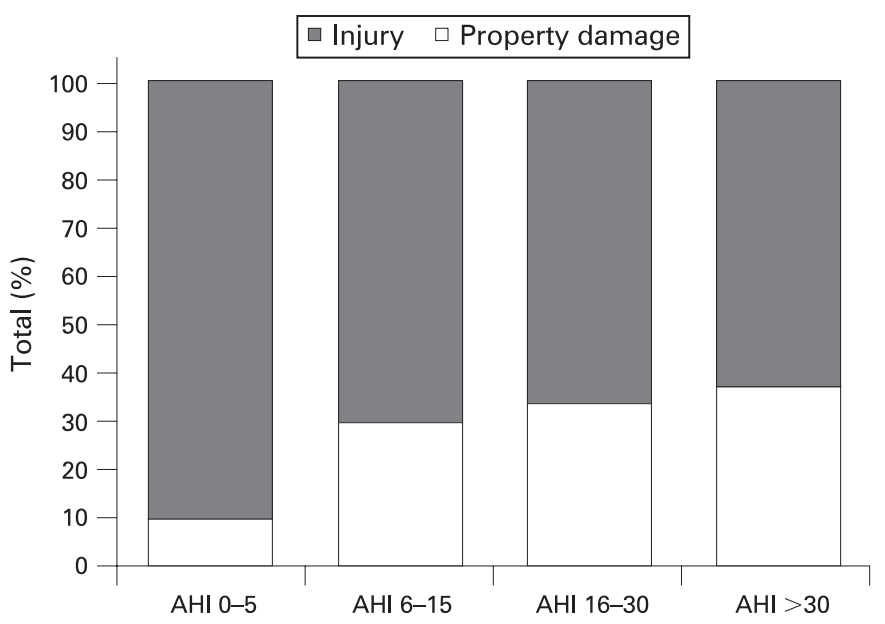

Figure 3 Proportion of crashes with injury and crashes causing property damage only for the patient group. AHI, apnoea-hypopnoea index. 


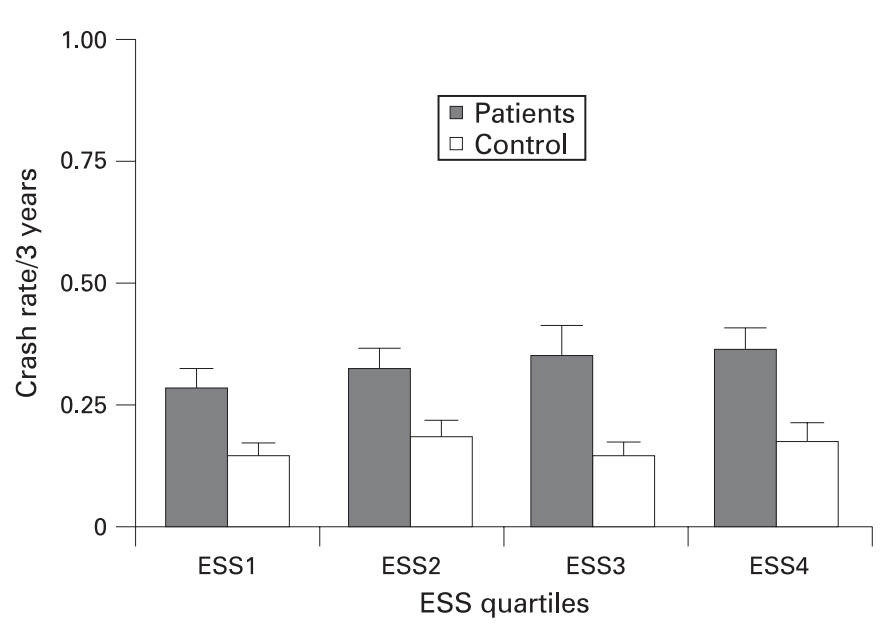

Figure 4 Mean crash rates over 3-year study period for patients separated into quartiles of subjective sleepiness based on the Epworth Sleepiness Scale (ESS). Error bars represent standard error of the mean.

have an "equalising" effect between the sexes in terms of driving risk.

We did not find a significant relationship between subjective sleepiness and rates of MVC. This is somewhat surprising given the findings in a recent large naturalistic driving study ${ }^{5}$ which indicated that drowsiness was a contributing factor in $10 \%$ of all crashes and $12 \%$ of near crashes. However, our study is consistent with other studies involving patients with OSAH. In a recent systematic review of papers examining rates of MVC in patients with OSAH, ESS was not predictive of MVC in 8 of 15 studies. ${ }^{27}$ Several factors may contribute to this counterintuitive finding. First, ESS is not a good predictor of OSAH severity and correlates poorly with multiple sleep latency testing. ${ }^{26}$ Second, patients who score highly on the ESS are by definition aware of their propensity to fall asleep and thus have potential to adapt their behaviour accordingly. Patients who are less aware of impairment may be at much higher risk. This possibility was underlined in a study by Connor et $a{ }^{24}{ }^{24}$ where acute sleepiness measured on the Stanford Sleepiness Scale ${ }^{28}$ was associated with increased risk of death or injury in MVCs but chronic sleepiness as determined by the ESS was not, underlining the possibility of adaptive strategies by drivers who are aware of their own levels of sleepiness. We suspect that many patients with OSAH may not be effective in assessing their impairment and that this confers increased risk. Third, patients may intentionally misrepresent their levels of sleepiness because of concerns related to exclusion from driving. Fourth, our study is a retrospective analysis of a referral population as opposed to a population-based study. As such, it may be possible that patients may be referred because they perceive a problem with their driving (whether or not they complain of sleepiness per se), thus minimising the relationship between subjective sleepiness and crash risk. Future prospective studies may clarify this issue. Fifth, for many of the variables (eg, caffeine use, kilometres driven) we have relied on self-reports of patients. For some of these, self-reporting may be inaccurate (eg, sedatives, coffee) which might be a potential explanation for the lack of a significant association between these variables and MVCs.

Driving is a complex task, and driving performance is subject to a wider range of influences. The relationship between OSAH severity, sleepiness and the ability to perform intricate tasks shows considerable variability. ${ }^{29}$ Simulated driving performance is certainly impaired by OSAH and improved by continuous positive airway pressure, ${ }^{30-32}$ but it is unclear whether simulators can be used to gauge MVC risk. ${ }^{33}{ }^{34}$ In addition, our data indicate that the increased risk of MVC occurs at all levels of OSAH severity, confirming the findings of Barbe and colleagues ${ }^{4}$ that conventional markers of OSAH severity do not discriminate those patients at higher risk of MVC. In any event, caution should be exercised when assessing patients' driving risk, and all patients with OSAH should be advised of the potential risk.

Our study had a number of strengths compared with previous studies in this area. First, this is the largest study of this kind to date with a large range of OSAH severity. Second, we used a validated measure of MVC rates (provincial insurance records) rather than self-reports. Third, we collected information about a number of potential confounders including use of alcohol, sedatives and stimulants. Finally, our study is the first to examine the relationship between MVC severity and OSAH and confirms directly the figures reported by George. ${ }^{35}$ However, our study also had limitations. First, it was retrospective in design in that PSG information was collected after the MVC. We therefore cannot exclude the possibility that the occurrence of

Table 3 Multivariable Poisson regression modelling

\begin{tabular}{|c|c|c|c|c|}
\hline & \multicolumn{2}{|l|}{ Unselected MVCs } & \multicolumn{2}{|l|}{ MVCs with injury } \\
\hline & Rate ratio (95\% Cl) & p Value & Rate ratio $(95 \% \mathrm{CI})$ & p Value \\
\hline \multicolumn{5}{|l|}{ Patients } \\
\hline Presence of OSAH & 1.22 (0.92 to 2.01$)$ & 0.12 & 3.67 (1.30 to 10.3$)$ & 0.014 \\
\hline ESS & 1.01 (0.99 to 1.04$)$ & 0.35 & 1.04 (0.99 to 1.09$)$ & 0.11 \\
\hline Male sex & 0.91 (0.66 to 1.25$)$ & 0.55 & 0.59 (0.32 to 1.09$)$ & 0.09 \\
\hline Age & $1.00(0.99$ to 1.01$)$ & 0.69 & $0.99(0.97$ to 1.02$)$ & 0.50 \\
\hline BMl & 1.01 (1.00 to 1.02$)$ & 0.008 & $0.99(0.96$ to 1.03$)$ & 0.69 \\
\hline Per 100 km driven/week & 1.09 (1.03 to 1.16$)$ & 0.003 & $1.12(1.00$ to 1.25$)$ & 0.053 \\
\hline Alcohol use* & $1.01(1.00$ to 1.03$)$ & 0.12 & 1.05 (1.02 to 1.07$)$ & 0.0014 \\
\hline Sedative use & 0.79 (0.49 to 1.28$)$ & 0.34 & $0.96(0.41$ to 2.23$)$ & 0.92 \\
\hline Caffeine use $\dagger$ & 0.99 (0.98 to 1.00$)$ & 0.15 & 0.99 (0.5 to 1.3$)$ & 0.19 \\
\hline \multicolumn{5}{|l|}{ Controls } \\
\hline Male sex & 1.55 (1.00 to 2.41$)$ & 0.049 & $2.39(0.71$ to 8.08$)$ & 0.13 \\
\hline Age & 0.99 (0.98 to 1.01$)$ & 0.38 & 0.95 (0.92 to 0.99$)$ & 0.006 \\
\hline
\end{tabular}

BMI, body mass index; ESS, Epworth Sleepiness Scale; MVC, motor vehicle crash; OSAH, obstructive sleep apnoea/hypopnoea. ${ }^{*}$ For every one increase in alcohol use per week (ie, bottle/can/glass of beer, 4 oz wine or drink/shot of liquor).

$\dagger$ †or every one cup increase in coffee use per week. 
MVC was a reason for referral to the sleep laboratory. This could have artificially increased the difference between the rates in controls and patients, and may be a potential explanation for the slightly increased rate of MVC in the 0-5 AHI group compared with controls (odds ratio 1.5) that was nearly significant. However, this should not have affected the relative risk between patients with and without OSAH. Second, our study was based on patients referred to a sleep disorder clinic with suspected sleep-disordered breathing so our results may not be applicable outside this type of patient population. Patients may have been partially referred on the basis of sleepiness, and this could help explain the increased rate of MVCs in our patients who did not have OSAH (ie, they had causes of sleepiness other than OSAH). This could also explain the relatively poor correlation between AHI and ESS in our study $(r=0.13, p<0.001)$.

In summary, patients with OSAH have an increased rate of MVC compared with patients without OSAH and age- and sexmatched controls. Patients with OSAH are particularly prone to MVCs associated with personal injury, and this increased risk occurs even in patients with mild OSAH. Subjective daytime sleepiness does not appear to be particularly helpful in terms of risk prediction for MVC, which indicates a need for alternative strategies to assess driving performance. ${ }^{36}{ }^{37}$ Treatment with continuous positive airway pressure has been shown to normalise crash rates ${ }^{35}$ and improve vigilance ${ }^{38}$ in patients with OSAH. MVCs associated with OSAH are potentially preventable. Increasing public awareness of the symptoms and risks of OSAH together with improved access to diagnosis and treatment is likely to confer major cost savings to society in addition to benefiting individual patients. ${ }^{39-41}$

Funding: This research was funded by a Michael Smith Foundation for Health Research Unit award and a British Columbia Lung Association operating grant. AM is supported by a CIHR/BC Lung Fellowship and by the CIHR/HSFC IMPACT training scheme. NA is supported by a Michael Smith Foundation for Health Research Scholar Award, a CIHR/BC Lung Association New Investigator Award and a Departmental Scholar Award from the University British Columbia.

Competing interests: None.

Ethics approval: The study was approved by the university ethics board and all patients provided written informed consent.

\section{REFERENCES}

1. George CF, Nickerson PW, Hanly PJ, et al. Sleep apnoea patients have more automobile accidents. Lancet 1987;2:447.

2. Findley LJ, Unverzagt ME, Suratt PM. Automobile accidents involving patients with obstructive sleep apnea. Am Rev Respir Dis 1988;138:337-40.

3. George CF, Smiley A. Sleep apnea and automobile crashes. Sleep 1999;22:790-5.

4. Barbe F, Pericas J, Munoz A, et al. Automobile accidents in patients with sleep apnea syndrome. An epidemiological and mechanistic study. Am J Respir Crit Care Med 1998;158:18-22.

5. Dingus TA, Klauer SG, Neale VL, et al. The 100-Car Naturalistic Driving Study. National Highway Traffic Safety Administration. Report DOT HS 810 593, 2006.

6. Horstmann S, Hess CW, Bassetti C, et al. Sleepiness-related accidents in sleep apnea patients. Sleep 2000;23:383-9.

7. Teran-Santos J, Jimenez-Gomez A, Cordero-Guevara J. The association between sleep apnea and the risk of traffic accidents. Cooperative Group Burgos-Santander. N Engl J Med 1999;340:847-51

8. Young T, Blustein J, Finn L, et al. Sleep-disordered breathing and motor vehicle accidents in a population-based sample of employed adults. Sleep 1997;20:608-13.

9. Haraldsson P0, Carenfelt C, Diderichsen F, et al. Clinical symptoms of sleep apnea syndrome and automobile accidents. ORL J Otorhinolaryngol Relat Spec 1990;52:5762.

10. Aldrich MS. Automobile accidents in patients with sleep disorders. Sleep 1989;12:487-94.

11. Findley L, Unverzagt $\mathrm{M}$, Guchu $\mathrm{R}$, et al. Vigilance and automobile accidents in patients with sleep apnea or narcolepsy. Chest 1995;108:619-24.
12. Masa JF, Rubio M, Findley LJ. Habitually sleepy drivers have a high frequency of automobile crashes associated with respiratory disorders during sleep. Am J Respir Crit Care Med 2000;162:1407-12.

13. Lloberes $\mathbf{P}$, Levy G, Descals $\mathrm{C}$, et al. Self-reported sleepiness while driving as a risk factor for traffic accidents in patients with obstructive sleep apnoea syndrome and in non-apnoeic snorers. Respir Med 2000;94:971-6.

14. Noda A, Yagi T, Yokota M, et al. Daytime sleepiness and automobile accidents in patients with obstructive sleep apnea syndrome. Psychiatry Clin Neurosci 1998; 52:221-2.

15. Shiomi T, Arita AT, Sasanabe R, et al. Falling asleep while driving and automobile accidents among patients with obstructive sleep apnea-hypopnea syndrome. Psychiatry Clin Neurosci 2002:56:333-4.

16. Goncalves MA, Paiva T, Ramos E, et al. Obstructive sleep apnea syndrome, sleepiness, and quality of life. Chest 2004;125:2091-6.

17. Wu H, Yan-Go F. Self-reported automobile accidents involving patients with obstructive sleep apnea. Neurology 1996;46:1254-7.

18. Powell NB, Riley RW, Schechtman KB, et al. A comparative model: reaction time performance in sleep-disordered breathing versus alcohol-impaired controls. Laryngoscope 1999;109:1648-54.

19. Pack Al, Pack AM, Rodgman E, et al. Characteristics of crashes attributed to the driver having fallen asleep. Accid Anal Prev 1995;27:769-75.

20. Volna J, Sonka K. Medical factors of falling asleep behind the wheel. Prague Med Rep 2006:107:290-6.

21. McConnell CF, Bretz KM, Dwyer WO. Falling asleep at the wheel: a close look at 1,269 fatal and serious injury-producing crashes. Behav Sleep Med 2003;1:171-83.

22. Chervin RD, Aldrich MS, Pickett R, et al. Comparison of the results of the Epworth Sleepiness Scale and the Multiple Sleep Latency Test. J Psychosom Res 1997:42:145-55.

23. American Academy of Sleep Medicine Task Force. Sleep-related breathing disorders in adults: recommendations for syndrome definition and measurement techniques in clinical research. Sleep 1999;22:667-89.

24. Connor J, Norton R, Ameratunga $S$, et al. Driver sleepiness and risk of serious injury to car occupants: population based case control study. BMJ 2002;324:1125.

25. Vgontzas AN, Papanicolaou DA, Bixler EO, et al. Sleep apnea and daytime sleepiness and fatigue: relation to visceral obesity, insulin resistance, and hypercytokinemia. J Community Epidemiol Med 2000;85:1151-8.

26. Fong SY, Ho CK, Wing YK. Comparing MSLT and ESS in the measurement of excessive daytime sleepiness in obstructive sleep apnoea syndrome. J Psychosom Res 2005;58:55-60.

27. Ellen RM, Palayew SM, Molnar F, et al. Systematic review of motor vehicle crash risk in persons with sleep apnea. J Clin Sleep Med 2006;2:193-200.

28. Hoddes E, Zarcone V, Smythe $\mathrm{H}$, et al. Quantification of sleepiness: a new approach. Psychophysiology 1973;10:431-6.

29. Mulgrew AT, Ryan CF, Fleetham JA, et al. The impact of obstructive sleep apnea and daytime sleepiness on work limitation. Sleep Med 2007;9:42-53.

30. Hack MA, Choi SJ, Vijayapalan P, et al. Comparison of the effects of sleep deprivation, alcohol and obstructive sleep apnoea (OSA) on simulated steering performance. Respir Med 2001;95:594-601.

31. Hack M, Davies RJ, Mullins R, et al. Randomised prospective parallel trial of therapeutic versus subtherapeutic nasal continuous positive airway pressure on simulated steering performance in patients with obstructive sleep apnoea. Thorax 2000:55:224-31.

32. Findley LJ, Fabrizio MJ, Knight $\mathrm{H}$, et al. Driving simulator performance in patients with sleep apnea. Am Rev Respir Dis 1989;140:529-30.

33. Findley LJ, Levinson MP, Bonnie RJ. Driving performance and automobile accidents in patients with sleep apnea. Clin Chest Med 1992;13:427-35.

34. Turkington PM, Sircar M, Allgar V, et al. Relationship between obstructive sleep apnoea, driving simulator performance, and risk of road traffic accidents. Thorax 2001;:56:800-5

35. George CF. Reduction in motor vehicle collisions following treatment of sleep apnoea with nasal CPAP. Thorax 2001;56:508-12.

36. Turkington PM, Sircar M, Saralaya D, et al. Time course of changes in driving simulator performance with and without treatment in patients with sleep apnoea hypopnoea syndrome. Thorax 2004;59:56-9.

37. Orth M, Duchna HW, Leidag M, et al. Driving simulator and neuropsychological [corrected] testing in OSAS before and under CPAP therapy. Eur Respir $J$ 2005;26:898-903.

38. Patel SR, White DP, Malhotra A, et al. Continuous positive airway pressure therapy for treating sleepiness in a diverse population with obstructive sleep apnea: results of a meta-analysis. Arch Intern Med 2003;163:565-71.

39. Pack Al, Pien GW. How much do crashes related to obstructive sleep apnea cost? Sleep 2004;27:369-70.

40. Ayas NT, FitzGerald JM, Fleetham JA, et al. Cost-effectiveness of continuous positive airway pressure therapy for moderate to severe obstructive sleep apnea/ hypopnea. Arch Intern Med 2006;166:977-84.

41. Mulgrew AT, Fox N, Ayas NT, et al. Diagnosis and initial management of obstructive sleep apnea without polysomnography: a randomized validation study. Ann Intern Med 2007;146:157-66. 